\title{
5.5 REMOTE SENSING OF THE ATMOSPHERIC AEROSOLS AND NOCTILUCENT CLOUDS FROM SPACE
}

\author{
O. Avaste
}

Tartu State University, USSR

202444 Tartu, Estonia, USSR

\section{Gadsden}

University of Aberdeen, Aberdeen, AB9 2UE Scotland

\section{R. Room}

Laboratory of Space Radiometry, Institute of Astrophysics and Atmospheric Physics 202444 Tartu, Estonia, USSR

The method for the determination of the optical density of tropospheric and mesospheric aerosols and for the estimation of the scattering phase function in the forward directions $\left(0-30^{\circ}\right)$ is proposed. The method is based on measurements of the brightness of the twilight horizon with the high-resolution limb-camera having five separated fields of view. In some detail the features of the camera are described and the mathematical aspects of remote sensing data-inversion are discussed.
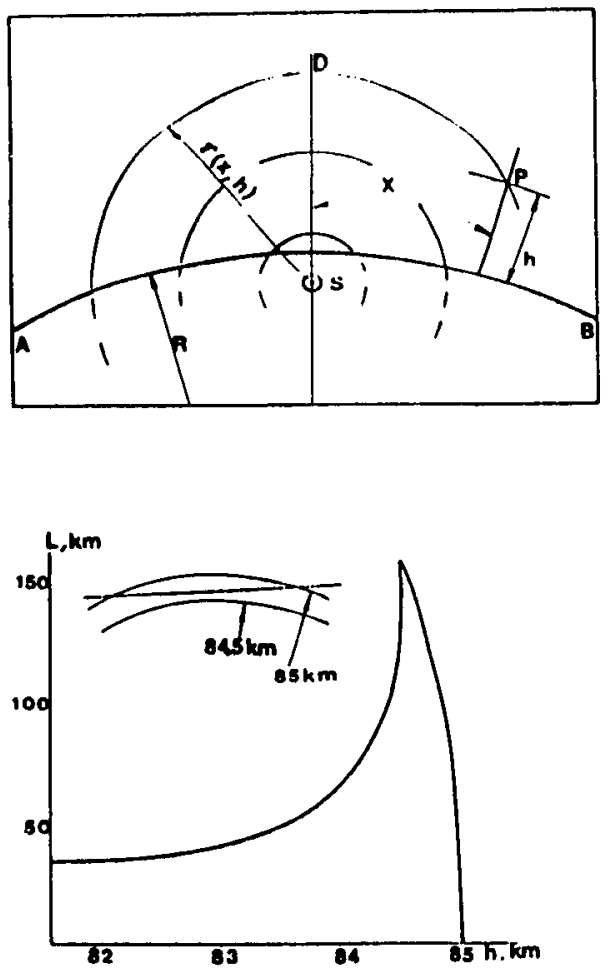

Figure 1. The coordinate system in the twilight horizon scanning equipment. AB - the limb of the Earth; $S$ - the center of the Sun; $P$ - the perigee point of the line-ofsight (line-of-sight is approximately perpendicular to the plane of figure).

Figure 2. The optical path through the homogeneous layer as a function of height. 